\title{
Duration of extracorporeal membrane oxygenation support and survival in cardiovascular surgery patients
}

\author{
Klaus Distelmaier, MD, PhD, ${ }^{\mathrm{a}, \mathrm{b}}$ Dominik Wiedemann, MD, ${ }^{\mathrm{b}, \mathrm{c}}$ Christina Binder, $\mathrm{MD},{ }^{\mathrm{a}, \mathrm{b}}$
} Thomas Haberl, MD, ${ }^{\mathrm{b}, \mathrm{c}}$ Daniel Zimpfer, MD,${ }^{\mathrm{b}, \mathrm{c}}$ Gottfried Heinz, MD, ${ }^{\mathrm{a}, \mathrm{b}}$ Herbert Koinig, MD, MBA, Alessia Felli, MD, ${ }^{\mathrm{e}}$ Barbara Steinlechner, MD, ${ }^{\mathrm{e}}$ Alexander Niessner, MD, MSc, ${ }^{\mathrm{a}, \mathrm{b}}$ Günther Laufer, MD, ${ }^{\mathrm{b}, \mathrm{c}}$ Irene M. Lang, $\mathrm{MD},{ }^{\mathrm{a}, \mathrm{b}}$ and Georg Goliasch, $\mathrm{MD}, \mathrm{PhD}^{\mathrm{a}, \mathrm{b}}$

\section{ABSTRACT}

Objective: The overall therapeutic goal of venoarterial extracorporeal membrane oxygenation (ECMO) in patients with postcardiotomy shock is bridging to myocardial recovery. However, in patients with irreversible myocardial damage prolonged ECMO treatment would cause a delay or even withholding of further permanent potentially life-saving therapeutic options. We therefore assessed the prognostic effect of duration of ECMO support on survival in adult patients after cardiovascular surgery.

Methods: We enrolled into our single-center registry a total of 354 patients who underwent venoarterial ECMO support after cardiovascular surgery at a university-affiliated tertiary care center.

Results: Through a median follow-up period of 45 months (interquartile range, 20-81 months), 245 patients $(69 \%)$ died. We observed an increase in mortality with increasing duration of ECMO support. The association between increased duration of ECMO support and mortality persisted in patients who survived ECMO support with a crude hazard ratio of 1.96 (95\% confidence interval, $1.40-2.74 ; P<.001$ ) for 2 -year mortality compared with the third tertile and the second tertile of ECMO duration. This effect was even more pronounced after multivariate adjustment using a bootstrap-selected confounder model with an adjusted hazard ratio of 2.30 (95\% confidence interval, $1.52-3.48 ; P<.001)$ for 2-year long-term mortality.

Conclusions: Prolonged venoarterial ECMO support is associated with poor outcome in adult patients after cardiovascular surgery. Our data suggest reevaluation of therapeutic strategies after 7 days of ECMO support because mortality disproportionally increases afterward. (J Thorac Cardiovasc Surg 2018;155:2471-6)

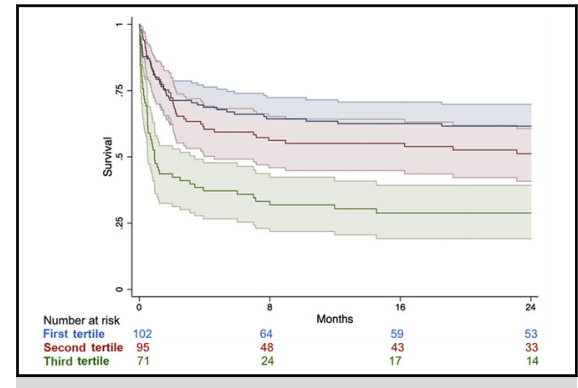

KM estimates of 2-year mortality according to tertiles of ECMO.

\section{Central Message}

Prolonged venoarterial ECMO support is associated with poor outcome in adult patients after cardiovascular surgery.

\section{Perspective}

The therapeutic goal of ECMO in patients with postcardiotomy shock is bridging to myocardial recovery. In patients with irreversible myocardial damage prolonged ECMO treatment would cause a delay of further potentially life-saving therapeutic options. Our data suggest timely reevaluation of strategies after 7 days of ECMO because mortality disproportionally increases afterward.

See Editorial Commentary page 2477

\footnotetext{
From the Departments of ${ }^{\mathrm{a}}$ Internal Medicine II, ${ }^{\mathrm{c}} \mathrm{Cardiac}$ Surgery, and ${ }^{\mathrm{e}}$ Cardiothoracic and Vascular Anaesthesia and Intensive Care Medicine, and ${ }^{\mathrm{b}} \mathrm{Center}$ for Cardiovascular Medicine, Medical University of Vienna, Vienna, Austria; and ${ }^{\mathrm{d}}$ University Hospital Krems, Department of Anaesthesia and Intensive Care Medicine, Karl Landsteiner University of Health Sciences, Krems, Austria.

This project was funded by the Medical Scientific Fund of the Mayor of the City of Vienna (2015; to K.D.)

Drs Distelmaier and Wiedemann contributed equally to this work.

Received for publication April 6, 2017; revisions received Oct 15, 2017; accepted for publication Dec 16, 2017; available ahead of print Feb 1, 2018.

Address for reprints: Dominik Wiedemann, MD, Department of Cardiac Surgery, Medical University of Vienna, Waehringer Guertel 18-20, A-1090 Vienna, Austria (E-mail: dominik.wiedemann@meduniwien.ac.at).

$0022-5223 / \$ 36.00$

Copyright (c) 2018 by The American Association for Thoracic Surgery

https://doi.org/10.1016/j.jtcvs.2017.12.079
}

Venoarterial extracorporeal membrane oxygenation (ECMO) might provide temporary cardiopulmonary support for patients with severe heart or lung failure after cardiovascular surgery. Although ECMO implantation for this indication is rapidly evolving, ${ }^{1}$ survival remains poor. ${ }^{2,3} \mathrm{~A}$ timely selection of patients in need of ECMO support and a prompt ECMO implantation by an experienced ECMO

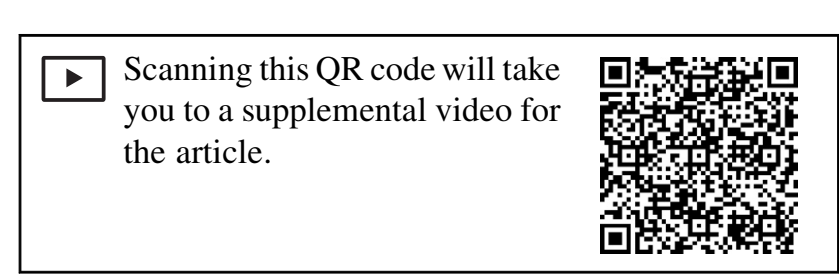




$$
\begin{aligned}
& \text { Abbreviations and Acronyms } \\
& \begin{aligned}
\text { CABG } & =\text { coronary artery bypass graft } \\
\text { CI } & =\text { confidence interval } \\
\text { ECMO } & =\text { extracorporeal membrane oxygenation } \\
\mathrm{HR} & =\text { hazard ratio } \\
\mathrm{IQR} & =\text { interquartile range } \\
\mathrm{VAD} & =\text { ventricular assist device }
\end{aligned}
\end{aligned}
$$

team is critical for patient outcome. ${ }^{4}$ The overall therapeutic goal of ECMO therapy is bridging to myocardial recovery in patients with postcardiotomy shock. Weaning trials recommend daily evaluation of myocardial function using clinical, echocardiographic, and hemodynamic measures. ${ }^{5}$ However, in patients with irreversible myocardial damage prolonged ECMO treatment would cause a withholding of further permanent potentially life-saving therapeutic options, namely heart transplantation or ventricular assist devices (VADs). Interestingly, there are no data aiming to further define this figurative point of no return from which the prospect of myocardial recovery diminishes and mortality rises. A more profound understanding of the time course of ECMO support might directly affect the subsequent patient treatment. In this study, we intended to analyze the prognostic effect of duration of ECMO support on survival in adult patients after cardiovascular surgery.

\section{METHODS \\ Study Population}

Between September 2003 and June 2014 all adult patients who underwent venoarterial ECMO therapy after cardiovascular surgery were enrolled in our registry. ${ }^{6}$ Patients who underwent ECMO support at the time of left VAD implantation were excluded from this analysis. All patients were recruited at the Vienna General Hospital, a universityaffiliated tertiary care center. The study was approved by the ethics committee of the Medical University of Vienna and is in line with the Declaration of Helsinki.

\section{Clinical Definitions and End Points}

Clinical ECMO management has been previously published in detail. ${ }^{7}$ Representative movie of ECMO implantation is available (Video 1). Indications for ECMO implantation were clinical signs of cardiogenic shock, such as systolic arterial hypotension $(<80 \mathrm{~mm} \mathrm{Hg}$ ), and signs of end organ failure, anaerobic metabolism, and metabolic acidosis despite optimized supportive measures (ie, inotropes, fluids, and intra-aortic balloon pump). ECMO duration was defined as time starting with ECMO implantation and ending with system explantation or death during ECMO support. All-cause 30-day mortality was selected as a primary end point and allcause long-term mortality as a secondary end point. Mortality data were obtained by screening the national registry of death including screening for the cause of death.

\section{Statistical Methods}

Baseline characteristics were compared using Kruskal-Wallis test and $\chi^{2}$ test, as appropriate. Cox proportional hazard regression analysis was applied to assess the effect of ECMO duration on survival. ECMO duration was stratified into tertiles and results are presented as the hazard ratio (HR) for comparison of the third tertile with the second tertile and the discriminatory power assessed using the Harrel C-statistic. To account for potential confounders we used a stepwise bootstrap resampling procedure including all baseline variables (excluding Simplified Acute Physiology Score II and EuroSCORE) to identify best-fitting variables for the final multivariable Cox regression model. Five hundred repeats with a $P$ value of .1 for selection were performed and variables selected in $50 \%$ of all repeats were included in the final cofounder model (ie, age, C-reactive protein). To test for interactions between ECMO duration and all variables in the final model, we used Cox proportional hazard regression models with ECMO duration, a variable in question, and the interaction between both variables. The proportional hazards assumption was tested and satisfied in all cases using Schoenfeld residuals. Kaplan-Meier analysis was applied to evaluate the effect of ECMO duration on survival and compared using log-rank test. Binary logistic regression analysis was performed to identify risk factors for being in the third tertile of ECMO duration. All statistical analyses were computed using SPSS version 23 (IBM Corp, Armonk, NY) and STATA 11 (StataCorp, College Station, Tex).

\section{RESULTS \\ Baseline Characteristics}

Between September 2003 and June 2014 at total of 10,547 patients underwent cardiovascular surgery at the Medical University of Vienna. Venoarterial ECMO therapy after cardiovascular surgery was needed in 385 $(3.7 \%)$ patients. After exclusion of patients who underwent ECMO support at the time of left VAD implantation $(\mathrm{n}=31)$, we included a total of 354 consecutive patients with a median age of 65 years (interquartile range [IQR], 55-73). Valve surgery was performed in 110 patients, coronary artery bypass graft (CABG) surgery in 48 , combined CABG-valve surgery in 84 , cardiac transplantation in 68 , aortic reconstruction in 24 , and other cardiovascular surgeries in 19 patients. The median procedure duration was 6 (IQR, 4:05-8:28) hours in patients with valve surgery, 6:45 (IQR, 4:20-8:55) hours in patients with CABG surgery, 8:29 (IQR, 6:45-10:13) hours in combined CABGvalve surgery, 8 (IQR, 6:40-9:51) hours in patients with cardiac transplantation, 8:10 (IQR, 6:10-10:05) hours in patients with aortic reconstruction, and 8:30 (IQR, 5:10$9: 20$ ) hours in patients with other cardiovascular surgeries.

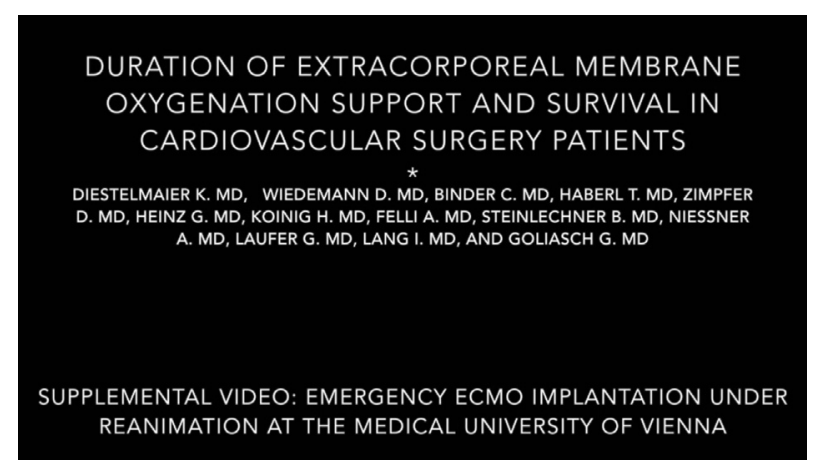

VIDEO 1. Video of emergency venoarterial extracorporeal membrane oxygenation implantation during cardiopulmonary resuscitation. Video available at: http://www.jtcvsonline.org/article/S0022-5223(17)33057-X/fulltext. 
TABLE 1. Baseline characteristics of total ECMO study population $(n=354)$ for tertiles of ECMO duration

\begin{tabular}{|c|c|c|c|c|c|}
\hline Parameter & $\begin{array}{l}\text { Total study population } \\
(\mathrm{n}=354)\end{array}$ & $\begin{array}{c}\text { First tertile } \\
(\mathrm{n}=144)\end{array}$ & $\begin{array}{l}\text { Second tertile } \\
(\mathrm{n}=111)\end{array}$ & $\begin{array}{c}\text { Third tertile } \\
(\mathbf{n}=99)\end{array}$ & $P$ value \\
\hline Tertiles of ECMO duration (IQR), $d$ & $4(2-7)$ & $2(1-3)$ & $5(4-6)$ & $9(7-13)$ & $<.001$ \\
\hline Age (IQR), y & $65(56-73)$ & $66(57-73)$ & $65(55-73)$ & $65(56-72)$ & .97 \\
\hline Body mass index (IQR), $\mathrm{n}$ & $27(24-30)$ & $27(24-30)$ & $27(24-31)$ & $27(24-30)$ & .75 \\
\hline Male sex, $\mathrm{n}(\%)$ & $243(69)$ & $97(67)$ & $81(73)$ & $65(66)$ & .48 \\
\hline SAPS II at ICU admission, n (\%) & $47(32-61)$ & $50(35-64)$ & $43(29-58)$ & $48(32-65)$ & .09 \\
\hline Additive EuroSCORE (IQR), points & $10(8-13)$ & $10(7-13)$ & $10(7-12)$ & $11(8-13)$ & .25 \\
\hline Procedure duration (IQR), h & $7.3(5.3-9.3)$ & $7.3(5.3-9.3)$ & $7.3(5.2-9.3)$ & $7.3(6.0-9.6)$ & .85 \\
\hline IABP, n $(\%)$ & $44(12)$ & $21(15)$ & $13(12)$ & $10(10)$ & .56 \\
\hline Hypertension, $\mathrm{n}(\%)$ & $241(72)$ & $96(69)$ & $75(71)$ & $70(75)$ & .59 \\
\hline Diabetes, n (\%) & $89(26)$ & $33(24)$ & $23(22)$ & $33(35)$ & .06 \\
\hline Hypercholesterolemia, n (\%) & $170(51)$ & $63(46)$ & $59(56)$ & $48(52)$ & .25 \\
\hline Coronary artery disease, $\mathrm{n}(\%)$ & $171(52)$ & $61(44)$ & $53(53)$ & $57(62)$ & .03 \\
\hline \multicolumn{6}{|l|}{ Left ventricular ejection fraction } \\
\hline $30 \%-44 \%, n(\%)$ & $53(16)$ & $26(19)$ & $13(13)$ & $14(15)$ & .41 \\
\hline$<30 \%, \mathrm{n}(\%)$ & $128(38)$ & $45(32)$ & $47(45)$ & $36(38)$ & .13 \\
\hline Glucose (IQR), mg/dL & $116(96-154)$ & $115(97-168)$ & $110(94-139)$ & $120(101-156)$ & .26 \\
\hline Total cholesterol (IQR), mg/dL & $143(106-182)$ & $144(107-188)$ & $144(108-185)$ & $139(105-171)$ & .78 \\
\hline Triglycerides (IQR), mg/dL & $109(78-161)$ & $114(79-167)$ & $102(74-158)$ & $111(80-161)$ & .81 \\
\hline Creatinine (IQR), mg/dL & $1.3(1.1-1.8)$ & $1.4(1.0-1.8)$ & $1.3(1.1-1.7)$ & $1.3(1.0-1.9)$ & .73 \\
\hline Estimated GFR(IQR), $\mathrm{mL} / \mathrm{min} / 1.73 \mathrm{~m}^{2}$ & $51(38-70)$ & $51(35-69)$ & $54(40-70)$ & $49(37-67)$ & .44 \\
\hline Blood urea nitrogen (IQR), mg/dL & $25(19-38)$ & $24(19-36)$ & $23(16-37)$ & $31(20-41)$ & .07 \\
\hline Total bilirubin (IQR), mg/dL & $0.9(0.6-1.5)$ & $0.9(0.6-1.5)$ & $1.1(0.7-1.7)$ & $0.9(0.6-1.5)$ & .16 \\
\hline ASAT (IQR), U/L & $38(25-80)$ & $40(25-80)$ & $37(23-102)$ & $37(28-70)$ & .92 \\
\hline ALAT (IQR), U/L & $29(19-52)$ & $27(19-49)$ & $29(17-50)$ & $30(19-61)$ & .74 \\
\hline Gamma-GT, U/L (IQR) & $54(30-109)$ & $50(28-109)$ & $54(31-97)$ & $63(35-135)$ & .19 \\
\hline Hemoglobin (IQR), mg/dL & $12.0(10.3-13.7)$ & $12(10.5-13.9)$ & $12.0(10.3-14.4)$ & $11.7(10.1-13.2)$ & .25 \\
\hline Platelets (IQR), $1000 / \mu \mathrm{L}$ & $189(134-243)$ & $195(144-241)$ & $189(134-248)$ & $184(132-240)$ & .82 \\
\hline C-reactive protein (IQR), $\mathrm{mg} / \mathrm{dL}$ & $1.0(0.4-5.5)$ & $1.0(0.4-6.0)$ & $0.9(0.4-7.2)$ & $1.3(0.3-4.4)$ & .95 \\
\hline Leukocytes (IQR), $1000 / \mu \mathrm{L}$ & $8.8(6.5-13.6)$ & $9.2(6.7-14.4)$ & $8.0(6.0-12.8)$ & $8.4(6.6-12.1)$ & .33 \\
\hline
\end{tabular}

Counts are given as numbers and percentages. Continuous variables are given as median and IQR. Boldface indicates statistical significance. ECMO, Extracorporeal membrane oxygenation; IQR, interquartile range; SAPS II, Simplified Acute Physiology Score II; ICU, intensive care unit; IABP, intra-aortic balloon pump; GFR, glomerular filtration rate estimated using Cockcroft-Gault formula; $A S A T$, aspartate aminotransferase; $A L A T$, alanine aminotransferase; $G T$, glutamyl transferase.

The median duration of ECMO therapy was 4 (IQR, 2-7) days. ECMO duration did not significantly differ between different types of surgery $(P=.30)$ and was 4 (IQR, 2-7) days in patients with valve surgery, 5 (IQR, 2-6) days in patients with CABG surgery, 5 (IQR, 3-7) days in combined CABG-valve surgery, 4 (IQR, 3-7) days in patients with cardiac transplantation, 4 (IQR, 2-7) days in patients with aortic reconstruction, and 5 (IQR, 1-9) days in patients with other cardiovascular surgeries. ECMO support was initiated because of failure of cardiopulmonary bypass weaning $(58 \%)$, postoperative cardiogenic shock $(24 \%)$, post-transplant cardiac graft failure $(5 \%)$, postoperative bleeding/tamponade with cardiogenic shock $(5 \%)$, and miscellaneous conditions $(8 \%)$. Detailed baseline characteristics are shown in Table 1.

\section{ECMO Duration and Outcome}

During a median follow-up time of 45 months (IQR, 2081 months), 245 patients (69\%) died. We observed an increase in mortality with increasing duration of ECMO support evidenced by Kaplan-Meier analysis as well as a steep increase in the cumulative hazards with increasing duration of ECMO support (Figure 1). The association between increased duration of ECMO support and mortality 

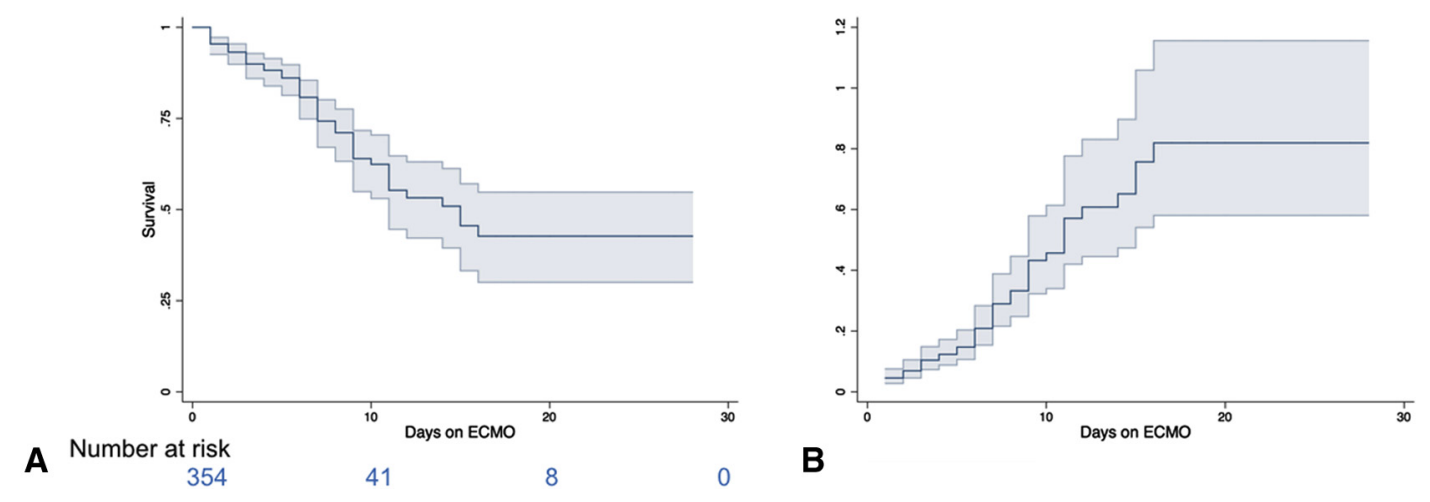

FIGURE 1. Time-related survival of patients receiving extracorporeal membrane oxygenation (ECMO); Kaplan-Meier (A) and cumulative hazard function (B) of patient survival with ECMO support.

persisted even in patients who survived ECMO support at 2 -year long-term follow-up $(P<.001$; Figure 2$)$. We identified a significant adverse association of ECMO duration on mortality in patients who survived ECMO support with a crude $\mathrm{HR}$ of 1.96 (95\% confidence interval [CI], $1.40-2.74 ; P<.001)$ and a C-statistic of 0.60 for 2 -year mortality in a comparison of the third tertile with the second tertile of ECMO duration. This effect was even more pronounced after adjustment for potential confounders with an adjusted $\mathrm{HR}$ of $2.30(95 \% \mathrm{CI}, 1.52-3.48$; $P<.001)$ and a C-statistic of 0.67 for 2-year long-term mortality (Table 2). Furthermore, to depict the survival of the strategy commencing with ECMO support, we performed a Kaplan-Meier analysis starting with the initiation of ECMO and extending up to 24 months and observed over the entire observation period a significant increase of mortality with increasing duration of ECMO support (Figure 3).

Diabetes (odds ratio, $1.85 ; 95 \% \mathrm{CI}, 1.10-3.10 ; P=.02$ ) and history of coronary artery disease (odds ratio, 1.79;

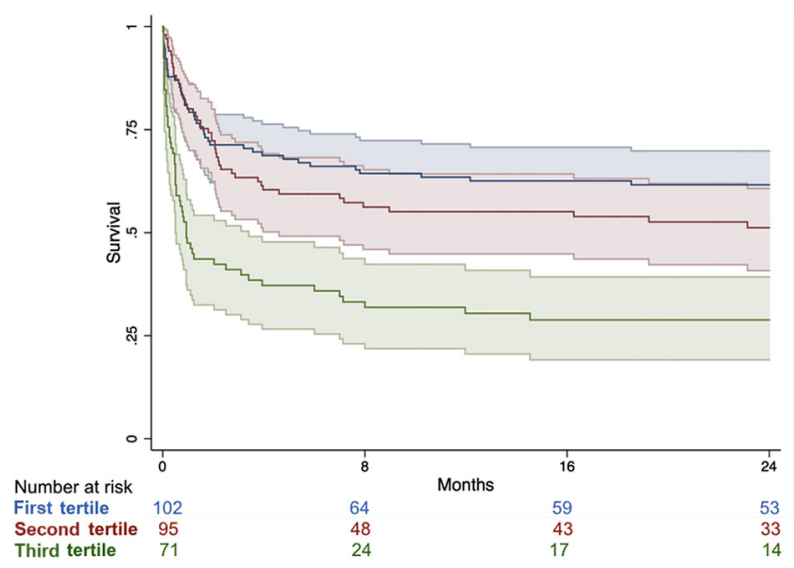

FIGURE 2. Kaplan-Meier estimates of mortality in extracorporeal membrane oxygenation (ECMO) survivors; Kaplan-Meier estimates of 2-year mortality long-term $(P<.001)$ in patients who survived ECMO support according to tertiles of ECMO duration.
$95 \%$ CI, 1.09-2.92; $P=.02$ ) were both significant risk factors for being within the third tertile of ECMO duration. Outcome subanalyses within the third tertile of ECMO duration revealed a significant association of age (crude HR, $1.28 ; 95 \% \mathrm{CI}, 1.03-1.61 ; P=.02$ ), right ventricular function as quantified using tricuspid annular plane systolic excursion (crude HR, 0.48 ; 95\% CI, $0.30-0.75 ; P=.001$ ), and urinary output within the first 24 hours of ECMO support (crude HR, 0.73; 95\% CI, 0.55-0.97; $P=.03$ ) with 2-year long-term prognosis, whereas no association was detected with sex $(P=.07)$, left ventricular function $(P=.83)$, Simplified Acute Physiology Score II at intensive care unit admission $(P=.52)$, and additive EuroSCORE $(P=.19)$.

During the observation period, 4 patients of the whole study population transitioned to VAD implantation and 3 patients underwent heart transplantation. These patients were significantly younger compared with the remaining study population (median age, 52 years; IQR, 23-54 vs 65 years; IQR, $56-72$ years; $P=.004$ ). We did not observe a beneficial effect of transition to durable ventricular assist options encompassing heart transplantation and left VAD for 30-day $(P=.19)$ or 2-year long-term $(P=.52)$ mortality compared with the residual study population. Interestingly, 4 of the 7 patients were within the third tertile of ECMO duration.

\section{DISCUSSION}

The present study reveals a strong and independent association between prolonged ECMO support and mortality in cardiovascular surgery patients. Although the prognosis of patients with short-term need of ECMO was promising, the outcome of patients requiring prolonged ECMO support was rather disenchanting.

Generally, venoarterial ECMO therapy aims to provide temporary mechanical circulatory support until myocardial recovery in patients with postcardiotomy shock to prevent tissue hypoperfusion and organ dysfunction. ${ }^{8}$ Daily weaning trials are recommended to minimize the duration of 
TABLE 2. Unadjusted and adjusted Cox proportional hazard model of ECMO duration for 2-year mortality in patients who survived ECMO support

\begin{tabular}{lcccccc}
\hline & Unadjusted HR $(\mathbf{9 5} \%$ CI) & $\boldsymbol{P}$ value & C-statistic & Adjusted HR (95\% CI) & $\boldsymbol{P}$ value & C-statistic \\
\hline ECMO duration, d & $1.96(1.40-2.74)$ & $<.001$ & 0.60 & $2.30(1.52-3.48)$ & $<.001$ & - \\
Age, y & $1.39(1.15-1.68)$ & $\mathbf{. 0 0 1}$ & 0.60 & $1.56(1.24-1.95)$ & $<.001$ & 0.67 \\
C-reactive protein, $\mathrm{mg} / \mathrm{dL}$ & $1.12(1.01-1.25)$ & $\mathbf{. 0 3 7}$ & 0.54 & $1.69(1.07-2.67)$ & $\mathbf{. 0 2 3}$ & - \\
\hline
\end{tabular}

HRs refer to a comparison of the third tertile with the second tertile of ECMO duration and a 1-SD increase in continuous variables. Boldface indicates statistical significance. $S D$, Standard deviation; $H R$, hazard ratio; $C I$, confidence interval; $E C M O$, extracorporeal membrane oxygenation.

ECMO support. ${ }^{9}$ However, the clinical course might vary considerably in patients with peri- or postoperative contractility impairment on the basis of the underlying pathophysiological mechanisms. Patients with transient postoperative myocardial stunning might stabilize quickly and get successfully weaned off ECMO, whereas patients with postcardiotomy cardiogenic shock caused by irreversible myocardial damage are unlikely to recover contractile function. ${ }^{10}$ Compounding their clinical situation, patients with prolonged ECMO support are highly vulnerable to ECMO complications including thromboembolic events, bleeding, infection, and end-organ dysfunction that significantly contribute to a high morbidity and mortality. Therefore, early identification of a poor capacity for myocardial recovery in ECMO patients is critical in the decision of transferring them timely to further potentially life-saving therapeutic options before the onset of complications that increase with extended ECMO duration. According to our data, this careful reevaluation of therapeutic management should be performed 7 days after ECMO implantation because further delay might seriously affect survival. Only 1 in 5 patients with prolonged need for ECMO support survive in the longer term. Considering the large proportion of patients requiring ECMO for more than 7 days ranging between $25 \%$ and $50 \%$ in current clinical practice, ${ }^{11,12}$ our study finding might have a profound effect on future

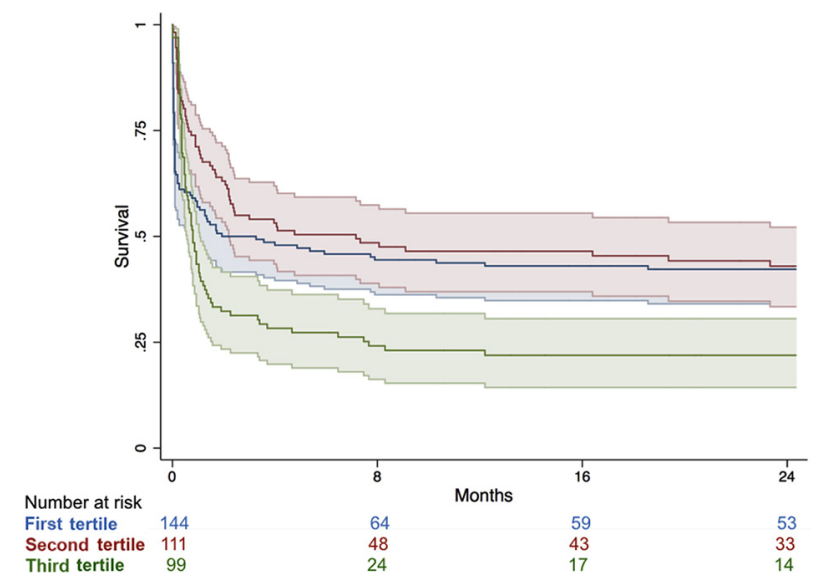

FIGURE 3. Kaplan-Meier estimates of mortality starting with the initiation of extracorporeal membrane oxygenation (ECMO); Kaplan-Meier estimates of 2-year mortality long-term $(P<.001)$ of all patients starting with initiation of ECMO according to tertiles of ECMO duration. patient care management. This observation is in conflict with previous data that recommended an early reevaluation of therapeutic strategies 48 to 72 hours after ECMO implantation. ${ }^{13}$ Improved ECMO techniques and the implementation of a multidisciplinary care approach, including an intensive care physician, a cardiac surgeon, and a perfusionist, might explain the extended safety profile of ECMO use over the past decade. ${ }^{7}$ Notably, even patients who were successfully weaned from prolonged ECMO support had a worse outcome compared with patients who were rapidly weaned. This observation supports the hypothesis that extracorporeal circulation per se might persistently trigger proinflammatory effects on the vasculature bed that might long-lastingly compromise patient recovery even after ECMO weaning. ${ }^{14}$

Additionally, a better characterization of the prolonged support group might aid the treating physician to reassess the clinical course and identify patients with prolonged ECMO support who might benefit from intensified therapies. A closer examination of patients belonging to the third tertile showed that these patients tended to have a higher incidence of diabetes and coronary artery disease. Outcome prediction of this highly vulnerable patient population was clearly limited by the use of established risk scores. However in accordance with previous studies, ${ }^{7,15}$ right ventricular function and urinary output within first 24 hours of ECMO support were confirmed as reliable predictors for clinical outcome.

On the basis of these data, the treating physician should be advised to timely evaluate further therapeutic strategies in patients with prolonged ECMO support, which are basically limited to 3 different options: first, forced ECMO weaning by the implementation of regular and standardized weaning trials in patients with suspected potential for recovery to keep ECMO support as short as possible. ${ }^{9}$ In this context, the current literature emphasizes the role of transesophageal echocardiography to guide a fast and safe ECMO weaning process. ${ }^{2,16}$ Second, in patients with refractory cardiogenic shock, transition to VAD or evaluation for urgent heart transplantation should be considered early. Although heart transplantation represents the treatment of choice in patients with advanced refractory heart failure, ${ }^{8}$ its application is frequently hindered by contraindications, donor shortage, and the resultant delay to transplantation, as well as the 
poor clinical condition of pretransplantation patients. Therefore, emergency VAD implantation is a viable therapeutic option in patients with critical advanced heart failure who are currently ineligible for urgent heart transplantation. ${ }^{8}$ Interestingly, in the present study, no survival benefit was observed for patients transitioned to a durable ventricular assist option, although their median age was significantly younger compared with the remaining study population. Because most of these patients (4 of 7) were within the third tertile of ECMO duration, it is tempting to speculate that the golden window of therapeutic escalation was already missed. Third, for patients who require prolonged ECMO who are not candidates for further therapies, a withdrawal of therapeutic support should be discussed to avoid unpromising interventions that might disproportionately increase hospital costs and resource consumption. An optimized and well defined management of these patients might even improve the quality of the remaining life for the patient, dependents, and the caregiver.

A potential limitation is that the study reflects a singlecenter experience, which might limit the generalizability of our results. However, patient management was not biased by one given regimen because enrollment was performed on various independent intensive care units including medical and surgery departments. Moreover, the evaluation for permanent therapeutic approaches in ECMO patients with refractory cardiogenic shock is restricted to tertiary care centers. Therefore, all ECMO patients have to be transferred to a tertiary care center on a timely basis, which is anyway recommended by current guidelines. ${ }^{8}$ Furthermore, the study population was highly heterogeneous, because it comprised nearly the entire spectrum of cardiovascular surgery. Another significant limitation of this study was the lack of predefined weaning trials to keep ECMO support in all patients as short as possible. Although standardized ECMO weaning protocols were established, we cannot exclude that prolonged ECMO support might be occasionally caused by cautiousness of the treating physician. Finally, not only survival data, but also information about the quality of life of ECMO survivors, which were not recorded, might be relevant to comprehensively assess clinical success.

\section{CONCLUSIONS}

The present study advances the limited knowledge on the timing when prolonged venoarterial ECMO treatment becomes futile or even harmful in adult cardiovascular surgery patients. Our data suggest reevaluation of further therapeutic strategies after 7 days of ECMO support because mortality disproportionally increases afterward.

\section{Conflict of Interest Statement}

Authors have nothing to disclose with regard to commercial support.

\section{References}

1. Paden ML, Conrad SA, Rycus PT, Thiagarajan RR, Registry E. Extracorporeal Life Support Organization Registry Report 2012. ASAIO J. 2013;59: 202-10.

2. Rastan AJ, Dege A, Mohr M, Doll N, Falk V, Walther T, et al. Early and late outcomes of 517 consecutive adult patients treated with extracorporeal membrane oxygenation for refractory postcardiotomy cardiogenic shock. J Thorac Cardiovasc Surg. 2010;139:302-11, 11.e1.

3. Combes A, Leprince P, Luyt CE, Bonnet N, Trouiellt JL, Léger P, et al. Outcomes and long-term quality-of-life of patients supported by extracorporeal membrane oxygenation for refractory cardiogenic shock. Crit Care Med. 2008;36:1404-11.

4. Takayama H, Truby L, Koekort M, Uriel N, Colombo P, Mancini DM, et al. Clinical outcome of mechanical circulatory support for refractory cardiogenic shock in the current era. J Heart Lung Transplant. 2013;32:106-11.

5. Aissaoui N, Luyt CE, Leprince P, Trouillet JL, Léger P, Pavie A, et al. Predictors of successful extracorporeal membrane oxygenation (ECMO) weaning after assistance for refractory cardiogenic shock. Intensive Care Med. 2011;37:1738-45.

6. Roth C, Schrutka L, Binder C, Kriechbaumer L, Heinz G, Lang IM, et al. Liver function predicts survival in patients undergoing extracorporeal membrane oxygenation following cardiovascular surgery. Crit Care. 2016;20:57.

7. Distelmaier K, Roth C, Binder C, Schrutka L, Schreiber C, Hoffelner F, et al. Urinary output predicts survival in patients undergoing extracorporeal membrane oxygenation following cardiovascular surgery. Crit Care Med. 2016;44:531-8.

8. Ponikowski P, Voors AA, Anker SD, Bueno H, Cleland JG, Coats AJ, et al. 2016 ESC guidelines for the diagnosis and treatment of acute and chronic heart failure: the Task Force for the diagnosis and treatment of acute and chronic heart failure of the European Society of Cardiology (ESC) developed with the special contribution of the Heart Failure Association (HFA) of the ESC. Eur Heart J. 2016;37: 2129-200.

9. Mueller C, Christ M, Cowie M, Cullen L, Maisel AS, Masip J, et al. European Society of Cardiology-Acute Cardiovascular Care Association position paper on acute heart failure: a call for interdisciplinary care. Eur Heart J Acute Cardiovasc Care. 2017;6:81-6.

10. Pennington DG, Farrar DJ, Loisance D, Pae WE Jr, Emery RW. Circulatory support 1991. The second international conference on circulatory support devices for severe heart failure. Patient selection. Ann Thorac Surg. 1993;55: 206-12.

11. Aubron C, Cheng AC, Pilcher D, Leong T, Magrin G, Cooper DJ, et al. Factors associated with outcomes of patients on extracorporeal membrane oxygenation support: a 5-year cohort study. Crit Care. 2013;17:R73.

12. Schmidt M, Burrell A, Roberts L, Bailey M, Sheldrake J, Rycus PT, et al. Predicting survival after ECMO for refractory cardiogenic shock: the survival after veno-arterial-ECMO (SAVE)-score. Eur Heart J. 2015;36:2246-56.

13. Fiser SM, Tribble CG, Kaza AK, Long SM, Zacour RK, Kern JA, et al. When to discontinue extracorporeal membrane oxygenation for postcardiotomy support. Ann Thorac Surg. 2001;71:210-4.

14. Millar JE, Fanning JP, McDonald CI, McAuley DF, Fraser JF. The inflammatory response to extracorporeal membrane oxygenation (ECMO): a review of the pathophysiology. Crit Care. 2016;20:387.

15. Bartko PE, Wiedemann D, Schrutka L, Binder C, Santos-Gallego CG, Zuckermann A, et al. Impact of right ventricular performance in patients undergoing extracorporeal membrane oxygenation following cardiac surgery. J Am Heart Assoc. 2017;6:e05455.

16. Cavarocchi NC, Pitcher HT, Yang Q, Karbowski P, Miessau J, Hastings HM, et al. Weaning of extracorporeal membrane oxygenation using continuous hemodynamic transesophageal echocardiography. J Thorac Cardiovasc Surg. 2013; 146:1474-9.

Key Words: extracorporeal membrane oxygenation, cardiovascular surgery, outcome, mortality 